M. Nakai

Nagoya Math. J.

Vol. 92 (1983). 163-173

\title{
THE CORONA PROBLEM ON FINITELY SHEETED COVERING SURFACES
}

\author{
MITSURU NAKAI \\ Dedicated to Professor Makoto Ohtsuka on the \\ occasion of his 60th birthday
}

The purpose of this paper is to show that the corona theorem is valid on any unbounded finitely sheeted covering surface if and only if the corona theorem is valid on its base surface.

We start by fixing terminologies precisely before stating our main theorem. We denote by $H^{\circ}(R)$ the class of all single valued bounded analytic functions on a Riemann surface $R$. We say that the corona theorem is valid on $R$ if for any finite number of functions $f_{1}, \cdots, f_{s}$ in $H^{\infty}(R)$ with $\inf _{R} \sum_{j=1}^{s}\left|f_{j}\right|>0$ there exist functions $g_{1}, \cdots, g_{s}$ in $H^{\infty}(R)$ with $\sum_{j=1}^{s} f_{j} g_{j}=1$ on $R$.

Consider two Riemann surfaces $R$ and $\tilde{R}$ and an analytic mapping $\pi$ of $\tilde{R}$ onto $R$. We say that $(\tilde{R}, R, \pi)$, or simply $\tilde{R}$, is a covering surface of $R$. The surface $R$ and $\pi$ are referred to as the base surface and the covering map of the covering surface $(\tilde{R}, R, \pi)$. We say that the covering surface $(\tilde{R}, R, \pi)$ is unbounded if for any curve $C$ on $R$ with its initial point $z$ and any $\tilde{z}$ in $\pi^{-1}(z)$ there exists a curve $\tilde{C}$ on $\tilde{R}$ with $\tilde{z}$ its initial point such that $\pi(\tilde{C})=C$. Let $z_{0}$ be in $R$ and $\tilde{z}_{0}$ in $\pi^{-1}\left(z_{0}\right)$. We can always find local parameters $\zeta$ and $\tilde{\zeta}$ about $z_{0}$ and $\tilde{z}_{0}$ respectively such that the local expression of the covering map $z=\pi(\tilde{z})$ takes the form $\zeta=\tilde{\zeta}^{m}$. Here the positive integer $m$ does not depend on the choice of local parameters $\zeta$ and $\tilde{\zeta}$. If $m>1$, then $\tilde{z}_{0}$ is referred to as a branch point of order $m-1$. For each $z$ in $R$ we let $\#\left(\pi^{-1}(z)\right)=\infty$ if the set $\pi^{-1}(z)$ is infinite and $\#\left(\pi^{-1}(z)\right)=n$ if the set $\pi^{-1}(z)$ consists of a finite $n$ number of points where a branch point of order $m-1$ is counted as $m$ points. When $(\tilde{R}, R, \pi)$ is unbounded,

Received October 8, 1982.

This work was partly supported by Grant-in-Aid for Scientific Research, No. 454027, Japanese Ministry of Education, Science and Culture. 


$$
\#\left(\pi^{-1}(z)\right)=\sup _{\zeta \in R} \#\left(\pi^{-1}(\zeta)\right)=n \in N \cup\{\infty\}
$$

for any $z$ in $R$, where $N$ is the set of all positive integers. If $n \in N$, then we say that $(\tilde{R}, R, \pi)$ is $n$ sheeted or more roughly finitely sheeted without referring to the specific $n$. By using these terminologies our principal result in this paper is stated as follows:

The Main Theorem. The corona theorem is valid on any unbounded finitely sheeted covering Riemann surface $\tilde{R}$ if and only if the corona theorem is valid on the base Riemann surface $R$ of $\tilde{R}$.

Instead of proving this theorem directly we consider maximal ideal spaces $M$ and $\tilde{M}$ for $H^{\infty}(R)$ and $H^{\circ}(\tilde{R})$ respectively and the natural projection $\tau: \tilde{M} \rightarrow M$, i.e. $\tau(\tilde{p})=p$ if $\tilde{p} \cap H^{\infty}(R)=p$. We will clarify the structure of the "covering space" $(\tilde{M}, M, \tau)$ as the covering theorem in No. 7 from which the above theorem follows at once. In No. 1, we state applications, meanings, generality, and an open question of or related to our main theorem. In Nos. 2-5 some fundamental results on the maximal ideal spaces are given. In No. 6 the integral dependance of $H^{\infty}(\tilde{R})$ on $H^{\infty}(R)$ is explained. In No. 7 , the covering space $(\tilde{M}, M, \tau)$ is introduced and the covering theorem is stated, the proof of which will be given in Nos. 8-12.

1. Before proceeding to the proof we pause here to state three corollaries of our main theorem and a remark on the necessity of finitely sheetedness with an open question.

a) Every plane region or Riemann surface thus far known on which the corona theorem is valid is obtained based upon the fundamental result of Carleson [3] (see also Gamelin [7] for an extremely simple proof) that the corona theorem is valid on the unit disk $\Delta:|z|<1$. Stout [15] proved that the corona theorem is valid for any finitely eonnected plane region, and more generally Gamelin [4] proved that the corona theorem is valid for any finite open Riemann surface. As is well known these surfaces are represented as unbounded finitely sheeted covering surfaces of the unit disk $\Delta$ (cf. e.g. Ahlfors [1]). Hence, again based upon the Carleson result, our theorem may be viewed as a generalization of StoutGamelin theorem.

b) Consider an unbounded 2 sheeted covering surface $\left(\Delta_{2}, \Delta, \pi\right)$ of $\Delta$. As a special case of our theorem, the corona theorem is valid on $\Delta_{2}$ 
since it is valid on $\Delta$. This result is first obtained by Hara [9]. Let $\left\{c_{n}\right\}$ be the sequence of projections of branch points in $\Delta_{2}$. By the Blaschke theorem it is easy to see that $\sum\left(1-\left|c_{n}\right|\right)=+\infty$ if and only if $H^{\circ}\left(\Delta_{2}\right)$ $=H^{\infty}(\Delta) \circ \pi$. In this case the corona theorem is trivially valid on $\Delta_{2}$. Suppose $\sum\left(1-\left|c_{n}\right|\right)<+\infty$ and let $B$ be the Blaschke product with $\left\{c_{n}\right\}$ its zero set. It is then easy to see that $H^{\infty}\left(\Delta_{2}\right)$ is an $\left(H^{\infty}(\Delta) \circ \pi\right)$-module with the base $\{1, \sqrt{B}\}$. From this it instantly follows that $H^{\infty}\left(\Delta_{2}\right)$ separates the points in $\Delta_{2}$. Making use of the above module expression of $H^{\infty}\left(\Delta_{2}\right)$, Hara [9] proved that the corona theorem is valid on $\Delta_{2}$ which is an important nontrivial example of Riemann surfaces of infinite genus on which the corona theorem is valid.

c) Consider a region $W=\Omega-\cup \bar{D}_{n}$ obtained from a plane region $\Omega$ on which the corona theorem is valid by removing a sequence $\left\{\bar{D}_{n}\right\}$ of mutually disjoint closed disks $\bar{D}_{n}$ in $\Omega$ such that $\overline{\cup \bar{D}_{n}}-\cup \bar{D}_{n}$ is contained in $\partial \Omega$, the boundary of $\Omega$. Behrens [2] proved that the corona theorem is valid on $W$ along with $\Omega$ if the radii of $\bar{D}_{n}$ converge to zero sufficiently rapidly. This was the first example of plane regions of infinite connectivity on which the corona theorem is valid. It is sufficient to take $\Omega=\Delta$. Let us consider an unbounded 2 sheeted covering surface $W_{2}$ of $W$ with the sequence $\left\{c_{n}\right\}$ of projections of branch points in $W_{2}$. In general a hyperbolic Riemann surface $R$ is referred to as being of Parreau-Widom type if $\sum g\left(p_{n}, p\right)<+\infty$ where $g(\cdot, p)$ is the Green's function on $R$ with its pole $p$ and $\left\{p_{n}\right\}$ is the totality of zeros $p_{n}$ of $\nabla g(\cdot, p)$ on $R-p$. The importance of being of Parreau-Widom type lies in the fact that $H^{\infty}(R)$ separates the points in $R$ (Widom [16, 17]). It is easy to see that if the centers of $\bar{D}_{n}$ converge to $\partial \Delta$ sufficiently rapidly, then $W$ is of Parreau-Widom type. If $\left\{c_{n}\right\}$ converges to the boundary $\partial W$ sufficiently rapidly, then $W_{2}$ is of Parreau-Widom type along with $W$ (cf. Segawa [12]; see also Selberg [13], Yamamura [18], Stanton [14]). The Riemann surface $W_{2}$ is an example of Riemann surfaces of ParreauWidom type with infinite genus and of infinite connectivity on which the corona theorem is valid. A similar example was given by Hara [8].

d) In the "only if"-part of our theorem, the finitely sheetedness of $\tilde{R}$ is essential for the validity of its statement. There exists an example given by $\mathrm{B}$. Cole of Riemann surface $B$ of infinite genus and infinite connectivity on which the corona theorem is invalid (see Gamelin [6]). Moreover this $B$ can be chosen in the class of surfaces of Parreau-Widom 
type ([10]). Let $\tilde{B}$ be the universal covering surface of $B$. Since $B$. hyperbolic and not finite, $\tilde{B}=\Delta$ and is an unbounded infinite covering surface of $B$. Although the corona theorem is valid on $\tilde{B}=\Delta$, it is invalid on $B$.

e) In the "if"-part of our theorem, the authorm believes that the finitely sheetedness of $R$ is essential for the validity of its statement. If we can show that the Cole example $B$ can be represented as an unbounded covering surface of $\Delta$, then $(B, \Delta)$ gives a counterexample.

2. We now start our main discussion. For an arbitrary Riemann surface $R$ the class $H^{\infty}(R)$ is a ring over the complex number field $C$ and in fact a normed ring equipped with the norm $\|f\|=\sup _{R}|f|$. We denote by $M$ the maximal ideal space of the normed ring $H^{\infty}(R)$. The Gelfand transform $\hat{f}$ of $f$ in $H^{\infty}(R)$ is a function on $M$ where the value $\hat{f}(p)$ at each $p$ in $M$ is given by

$$
f-\hat{f}(p) \in p .
$$

A net $\left(p_{\lambda}\right)$ in $M$ converges to $p$ in $M$ if the net $\left(\hat{f}\left(p_{\lambda}\right)\right)$ converges to $\hat{f}(p)$ for every $f$ in $H^{\infty}(R)$. By this topology, $M$ is a compact Hausdorff space and $\widehat{H^{\infty}(R)}=\left\{\hat{f} ; f \in H^{\infty}(R)\right\}$ is a subring of $C(M)$, the ring of continuous functions on $M$, separating the points in $M$. These follow from the Gelfand theory of normed rings (see e.g. Gamelin [5], Rudin [11], etc). We denote by $\iota$ the natural map of $R$ into $M$ defined by

$$
\hat{f}(\iota(z))=f(z) \quad\left((z, f) \in R \times H^{\infty}(R)\right) .
$$

In general $\iota: R \rightarrow M$ is a continuous mapping. The map $\iota: R \rightarrow M$ is injective if and only if $H^{\circ}(R)$ separates the points in $R$. Even in this case, we do not know whether $\iota(R)$ is open in $M$. If $R$ is of ParreauWidom type, then $H^{\infty}(R)$ separates the points in $R, \iota(R)$ is open in $M$, and $R$ is homeomorphic to $\iota(R)$ (Stanton [14]).

3. Let $A$ be a subset of $M$. In order that $p \in M-\bar{A}$ it is necessary and sufficient that there exist functions $f_{1}, \cdots, f_{m}$ in $p$ with the property $\inf _{A} \sum_{j=1}^{m}\left|\hat{f}_{j}\right|>0$.

Suppose there exist such functions $f_{1}, \cdots, f_{m}$ and yet $p \in \bar{A}$. Then there exists a net $\left(p_{\lambda}\right)$ in $A$ converging to $p$ so that $\sum_{j=1}^{m}\left|\hat{f}_{j}\left(p_{\lambda}\right)\right| \rightarrow$ $\sum_{j=1}^{m}\left|\hat{f}_{j}(p)\right|=0$ which contradicts the assumption $\inf _{A} \sum_{j=1}^{m}\left|\hat{f}_{j}\right|>0$.

Conversely suppose that $p \in M-\bar{A}$. For each $q$ in $\bar{A}$ there exists an $f$ in $H^{\infty}(R)$ with $\hat{f}(p) \neq \hat{f}(q)$. Let $F(\cdot, q)=2(\hat{f}(q)-\hat{f}(p))^{-1}(f-\hat{f}(p))$. 
Then $F(\cdot, q)$ belongs to $H^{\infty}(R)$ with $\hat{F}(p, q)=0$ and $\hat{F}(q, q)=2$. On setting $V(q)=\{r \in M ;|\hat{F}(r, q)|>1\}$ we have $\bigcup_{q \in \bar{A}} V(q) \supset \bar{A}$. Therefore there exist points $q_{1}, \cdots, q_{m}$ in $\bar{A}$ with $\bigcup_{j=1}^{m} V\left(q_{j}\right) \supset \bar{A}$, and the functions $f_{j}=F\left(\cdot, q_{j}\right)$ in $H^{\infty}(R)(j=1, \cdots, m)$ are required.

4. A point $p$ in $M$ is said to be free if $p \notin \overline{\imath(R)}$. Viewing the totality of free points in $M$ as the "corona" of the "sun" $\iota(\bar{R})$, the term "corona problem", "corona conjecture", "corona theorem", etc have been used: The corona theorem is valid on $R$ if and only if $M$ contains no free points.

Let $f_{1}, \cdots, f_{m}$ be in $H^{\infty}(R)$ with $\inf _{R} \sum_{j=1}^{m}\left|f_{i}\right|>0$. If the set $P=$ $\left\{\sum_{j=1}^{m} f_{j} g_{j} ; g_{1}, \cdots, g_{m} \in H^{\infty}(R)\right\}$ does not contain 1 , then $P$ is a proper ideal of $H^{\circ}(R)$ and a fortiori there exists a $p$ in $M$ with $P \subset p$. Since $\inf _{\iota(R)} \sum_{j=1}^{m}\left|\hat{f}_{j}\right|=\inf _{R} \sum_{j=1}^{m}\left|f_{j}\right|>0$ and $f_{1}, \cdots, f_{m} \in p$, No. 3 assures that $p$ is free.

Conversely assume the existence of free $p$ in $M$. By No. 3 there exist $f_{1}, \cdots, f_{m}$ in $p$ with $\inf _{R} \sum_{j=1}^{m}\left|f_{j}\right|=\inf _{\iota(R)} \sum_{j=1}^{m}\left|\hat{f}_{j}\right|>0$. Then there are no $g_{1}, \cdots, g_{m}$ in $H^{\infty}(R)$ with $\sum_{j=1}^{m} f_{j} g_{j}=1$ on $R$.

5. The following almost trivial simple fact will play one of the fundamentally important decisive role in our proof of the covering theorem given later:

The Separation Lemma. For any mutually distinct points $p_{1}, \cdots, p_{m}$ in $M$ there exists an $f$ in $H^{\infty}(R)$ such that $\hat{f}\left(p_{1}\right), \cdots, \hat{f}\left(p_{m}\right)$ are mutually different.

For any pair $(i, j)$ with $1 \leqq i<j \leqq m$, since $p_{i} \neq p_{j}$, there exists an $f_{i j}$ in $H^{\infty}(R)$ with $\hat{f}_{i j}\left(p_{i}\right) \neq \hat{f}_{i j}\left(p_{j}\right)$. For any pair $(\mu, \nu)$ with $1 \leqq \mu<\nu \leqq m$, set, with $C$ the complex plane,

$$
P_{\mu \nu}=\left\{\left(z_{i j}\right) \in C^{m(m-1) / 2} ; \sum_{i<j}^{1, \cdots, m}\left(\hat{f}_{i j}\left(p_{\mu}\right)-\hat{f}_{i j}\left(p_{\nu}\right)\right) z_{i j}=0\right\} .
$$

Since $P_{\mu \nu}$ is a hyperplane in $C^{m(m-1) / 2}$, there exists a point $\left(\lambda_{i j}\right)$ in

$$
C^{m(m-1) / 2}-\bigcup_{\mu<\nu}^{1, \cdots, m} P_{\mu \nu} .
$$

Then the function

$$
f=\sum_{i<j}^{1, \cdots, m} \lambda_{i j} f_{i j}
$$

is a required function in $H^{\infty}(R)$. 
6. Let $R$ and $\tilde{R}$ be Riemann surfaces such that $(\tilde{R}, R, \pi)$ is an unbounded $n$ sheeted covering surface, $n$ being a positive integer. We now consider the relationship between rings $H^{\infty}(\tilde{R})$ and $H^{\infty}(R)$ over $C$. Clearly $H^{\infty}(R) \circ \pi$ is a subring isomorphic to $H^{\infty}(R)$. We set

$$
\pi^{-1}(z)=\left\{z_{1}, z_{2}, \cdots, z_{n}\right\}
$$

for each $z$ in $R$ where a branch point of order $m-1$ is appearing $m$ times as $m$ points in (3). To each $f$ in $H^{\infty}(\tilde{R})$ consider $n$ functions $a_{1}, \cdots, a_{n}$ in $H^{\infty}(R)$ defined by

$$
(-1)^{k} a_{k}(z)=\sum_{i_{1}<\cdots<i_{k}}^{1, \cdots, n} f\left(z_{i_{1}}\right) \cdots f\left(z_{i_{k}}\right) \quad(k=1,2, \cdots, n) .
$$

These functions $a_{1}, \cdots, a_{n}$ will be referred to as a-functions of $f$. Let $S$ be the totality of solutions of the algebraic equation

$$
X^{n}+a_{1}(z) X^{n-1}+\cdots+a_{n-1}(z) X+a_{n}(z)=0
$$

of degree $n$. Then we have

$$
S=\left\{f\left(z_{1}\right), \cdots, f\left(z_{n}\right)\right\}
$$

where a solution of order $m$ is appearing $m$ times in (6) which corresponds to a branch point of order $m-1$ in (3). By observing

$$
a_{i}(z)=\left(a_{i} \circ \pi\right)\left(z_{j}\right) \quad(i, j=1, \cdots, n),
$$

the relation (5) with $X=f\left(z_{j}\right)$ implies that the relation

$$
f^{n}+\left(a_{1} \circ \pi\right) f^{n-1}+\cdots+\left(a_{n-1} \circ \pi\right) f+a_{n} \circ \pi=0
$$

is valid on $\tilde{R}$. This means that, in terms of the ring theory (see e.g. Zariski-Samuel [19], p. 256), the ring $H^{\infty}(\tilde{R})$ is integral over $H^{\infty}(R) \circ \pi$.

7. We still assume that $(\tilde{R}, R, \pi)$ is an unbounded finitely $n$ sheeted covering surface of $R$. Let $M$ and $\tilde{M}$ be maximal ideal spaces of $H^{\infty}(R)$ and $H^{\infty}(\tilde{R})$, respectively, and $\iota$ and $\tilde{\iota}$ be natural maps of $R$ and $\tilde{R}$ to $M$ and $\tilde{M}$, respectively. We define the projection $\tau: \tilde{M} \rightarrow M$ by

$$
\hat{f}(\tau(p))=\widehat{f \circ \pi}(p) \quad\left((p, f) \in \tilde{M} \times H^{\infty}(R)\right) .
$$

It is instantly seen that $\tau: \tilde{M} \rightarrow M$ is continuous. For each $q$ in $M$ we call $\tau^{-1}(q)$ the fiber over $q$. Clearly the following is a commutative diagram: 


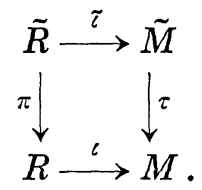

Concerning the structure of the covering space $(\tilde{M}, M, \tau)$, we have the following

The Covering Theorem. Let $(\tilde{R}, R, \pi)$ be an unbounded finitely $n$ sheeted covering surface of $R$ and $(\tilde{M}, M, \tau)$ the associated covering space. Then $\tau$ is surjective and the fiber $\tau^{-1}(p)$ over any $p$ in $M$ consists of at most $n$ points. Moreover

$$
\tau(\tilde{\imath}(\tilde{R}))=\iota(R), \quad \tilde{\imath}(\tilde{R})=\tau^{-1}(\iota(R))
$$

and also

$$
\tau(\overline{\imath(\tilde{R})})=\overline{\iota(\bar{R})}, \quad \overline{\tau(\tilde{R})}=\tau^{-1}(\overline{\iota(R)}) .
$$

In particular $\tilde{M}$ contains a free point if and only if $M$ contains a free point.

The main theorem follows at once from the above theorem with the result in No. 4. The proof of the above theorem will be given in Nos. 8-12 divided into several steps.

8. Here we start the proof of the covering theorem by showing that $\tau: \tilde{M} \rightarrow M$ is surjective, i.e. we have to show that $\tau^{-1}(p) \neq \emptyset$ for each $p$ in $M$. Consider the set

$$
P=\left\{\sum_{j=1}^{m} f_{j}\left(g_{j} \circ \pi\right) ; f_{j} \in H^{\infty}(\tilde{R}), g_{j} \in p, j=1, \cdots, m, m \in N\right\}
$$

which is an ideal in $H^{\infty}(\tilde{R})$. Suppose $P$ contains 1 . Then there exist functions $f_{j}$ in $H^{\infty}(\tilde{R})(j=1, \cdots, m)$ and $g_{j}$ in $p(j=1, \cdots, m)$ such that $\sum_{j=1}^{m} f_{j}\left(g_{j} \circ \pi\right)=1$. Let $\pi^{-1}(z)=\left\{z_{1}, \cdots, z_{n}\right\}$ for each $z$ in $R$ in the sense of (3). Since $g_{j} \circ \pi\left(z_{i}\right)=g_{j}(z)$, we have

$$
\sum_{j=1}^{m} f_{j}\left(z_{i}\right) g_{j}(z)=1 \quad(i=1, \cdots, n)
$$

Summing up these $n$ identities we obtain

$$
\sum_{j=1}^{m}\left(\sum_{i=1}^{n} f_{j}\left(z_{i}\right)\right) g_{j}(z)=n \text {. }
$$

If we set 


$$
h_{j}(z)=n^{-1} \sum_{i=1}^{n} f_{j}\left(z_{i}\right) \quad(j=1, \cdots, m),
$$

then $h_{j}$ belongs to $H^{\infty}(R)(j=1, \cdots, m)$ and $\sum_{j=1}^{m} h_{j}(z) g_{j}(z)=1$ for every $z$ in $R$. Hence $\sum_{j=1}^{m} \hat{h}_{j} \hat{g}_{j}=1$ on $M$ and in particular $\sum_{j=1}^{m} \hat{h}_{j}(p) \hat{g}_{j}(p)=1$. On the other hand $g_{j} \in p$ implies $\hat{g}_{j}(p)=0(j=1, \cdots, m)$ which contradicts the above identity. We have thus to conclude that $P$ is a proper ideal in $H^{\infty}(\tilde{R})$. Therefore there exists a $q$ in $\tilde{M}$ with $P \subset q$. The proof is over if we can show $\tau(q)=p$. Contrariwise suppose $\tau(q) \neq p$. Then there exists an $f$ in $H^{\infty}(R)$ such that $\hat{f}(\tau(q)) \neq \hat{f}(p)=0$. Then since $f$ belongs to $p, f \circ \pi$ belongs to $P$ and a fortiori to $q$. By (8), we see that $\hat{f}(\tau(q))=\widehat{f \circ \pi}(q)=0$, which contradicts the above.

9. Next we will show that the fiber $\tau^{-1}(p)$ consists of at most $n$ points for each point $p$ in $R$.

Contrary to the assertion we assume that the fiber $\tau^{-1}(p)$ contains $n+1$ different points $p_{0}, p_{1}, \cdots, p_{n}$ in $\tilde{M}$. By the separation lemma there exists an $f$ in $H^{\infty}(\tilde{R})$ such that $\hat{f}\left(p_{0}\right), \cdots, \hat{f}\left(p_{n}\right)$ are mutually different. Let $a_{1}, \cdots, a_{n}$ be the $a$-functions of $f$. Then from (7) it follows that

$$
\hat{f}\left(p_{j}\right)^{n}+\widehat{a_{1} \circ \pi}\left(p_{j}\right) \hat{f}\left(p_{j}\right)^{n-1}+\cdots+\widehat{a_{n-1} \circ \pi}\left(p_{j}\right) \hat{f}\left(p_{j}\right)+\widehat{a_{n} \circ \pi}\left(p_{j}\right)=0
$$

and by observing that $\widehat{a_{i} \circ \pi}\left(p_{j}\right)=\hat{a}_{i}\left(\tau\left(p_{j}\right)\right)=\hat{a}_{i}(p)(i=1, \cdots, n)$ we conclude that the algebraic equation

$$
X^{n}+\hat{a}_{1}(p) X^{n-1}+\cdots+\hat{a}_{n-1}(p) X+\hat{a}_{n}(p)=0
$$

of degree $n$ has $n+1$ different solutions $\hat{f}\left(p_{0}\right), \hat{f}\left(p_{1}\right), \cdots, \hat{f}\left(p_{n}\right)$, which is clearly a contradiction.

10. We proceed to the proof of (9). For the aim we only have to show that the inclusion relations $\tau(\tilde{\imath}(\tilde{R})) \subset \iota(R)$ and $\tau^{-1}(\iota(R)) \subset \tilde{\imath}(\tilde{R})$ are valid.

First let $z$ be an arbitrary point in $\tilde{R}$. By using (2) and (8) we deduce for $f$ in $H^{\infty}(R)$ that

$$
\hat{f}(\tau(\tilde{\imath}(z)))=\widehat{f \circ \pi}(\tilde{\iota}(z))=f \circ \pi(z)=f(\pi(z))=\hat{f}(\iota(\pi(z))) .
$$

Thus $\tau(\tilde{\imath}(z))=\iota(\pi(z)) \in \iota(R)$ and we have shown the inclusion relation $\tau(\tilde{\imath}(\tilde{R})) \subset \iota(R)$.

Conversely let $z$ be an arbitrary point in $R$. We will derive a contradiction from an erroneous assumption that there exists a point $p$ in $\tau^{-1}(\iota(z))$ which does not belong to $\tilde{\imath}(\tilde{R})$. Let $\pi^{-1}(z)=\left\{z_{1}, \cdots, z_{n}\right\}$ be as in 
(3). Observe that $\tau\left(\tilde{\imath}\left(\boldsymbol{z}_{i}\right)\right)=\iota\left(\pi\left(\boldsymbol{z}_{i}\right)\right)=\iota(z)$ as above. Hence $\left\{\tilde{\imath}\left(\boldsymbol{z}_{1}\right), \cdots, \tilde{\imath}\left(\boldsymbol{z}_{n}\right)\right\}$ $\subset \tau^{-1}(\iota(z))$ and $\tilde{\imath}(\tilde{R})$. Therefore $p \notin\left\{\tilde{\imath}\left(z_{1}\right), \cdots, \tilde{\imath}\left(z_{n}\right)\right\}$. By the separation lemma there exists an $f$ in $H^{\infty}(\tilde{R})$ with $\hat{f}(p) \notin\left\{f\left(z_{1}\right), \cdots, f\left(z_{n}\right)\right\}$. Let $a_{1}, \cdots, a_{n}$ be the $a$-functions of $f$. By considering Gelfand transforms of (7) at $p$ and by observing $\widehat{a_{i} \circ \pi}(p)=\hat{a}_{i}(\tau(p))=\hat{a}_{i}(\iota(z))=a_{i}(z)(i=1, \cdots, n)$, we conclude that $\hat{f}(p)$ is a solution of the algebraic equation

$$
X^{n}+a_{1}(z) X^{n-1}+\cdots+a_{n-1}(z) X+a_{n}(z)=0
$$

of degree $n$. On the other hand, by (6), the totality $S$ of solutions of the above equation is given by $S=\left\{f\left(z_{1}\right), \cdots, f\left(z_{n}\right)\right\}$ which does not contain $\hat{f}(p)$, a contradiction.

11. We turn to the proof of (10). As in No. 10 we only have to show that the inclusions $\tau\left(\overline{(\tilde{\imath}(\tilde{R}))} \subset \overline{\imath(R)}\right.$ and $\tau^{-1}(\overline{\iota(\bar{R})}) \subset \overline{\tau(\tilde{R})}$ are valid. Since, by $(9), \tau(\tilde{\imath}(\tilde{R})) \subset \iota(R)$, the continuity of $\tau$ implies at once that $\tau(\overline{\imath(\tilde{R})}) \subset \overline{\iota(R)}$. Thus we have to show that $\tau^{-1}(\overline{\iota(R)}) \subset \overline{\tau(\tilde{R})}$.

Choose an arbitrary $p$ in $\overline{\iota(R)}$ and then an arbitrary $q$ in $\tau^{-1}(p)$. We will show that $q$ belongs to $\bar{\imath}(\tilde{R})$. Choose a net $\left(z_{\lambda}\right)$ in $R$ such that $\left(\iota\left(z_{\lambda}\right)\right)$ converges to $p$ in $M$. Let

$$
\pi^{-1}\left(z_{\lambda}\right)=\left\{z_{1 \lambda}, \cdots, z_{n \lambda}\right\}
$$

in the sense of (3). Then we obtain $n$ nets $\left(\tilde{\imath}\left(z_{1 \lambda}\right)\right), \cdots,\left(\tilde{\imath}\left(z_{n \lambda}\right)\right)$ in $\tilde{M}$. Since $\tilde{M}$ is compact, there exists a subnet $\left(\tilde{c}\left(z_{1 \lambda^{\prime}}\right)\right)$ of $\left(\tilde{c}\left(z_{12}\right)\right)$ converging to a point $p_{1}$ in $\tilde{M}$. Similarly there exists a subnet $\left(\tilde{c}\left(z_{2 \lambda^{\prime \prime}}\right)\right)$ of $\left(\tilde{c}\left(z_{2 \lambda^{\prime}}\right)\right)$ converging to a point $p_{2}$ in $\tilde{M}$. By repeating the same process $n$ times we obtain a subnet $\left(\tilde{c}\left(\boldsymbol{z}_{n \lambda(n)}\right)\right)$ of $\left(\tilde{c}\left(\boldsymbol{z}_{n \lambda(n-1)}\right)\right)$ converging to a point $p_{n}$ in $\tilde{M}$. On replacing $(\lambda)$ by $\left(\lambda^{(n)}\right)$ if necessary, we can assume the following: $\left(\iota\left(z_{\lambda}\right)\right)$ converges to $p$ in $M$ and $\left(\tilde{\imath}\left(z_{i \lambda}\right)\right)$ converges to $p_{i}$ in $\tilde{M}(i=1, \cdots, n)$. Then we see that the continuity of $\tau$ implies that

$$
\tau\left(p_{i}\right)=\lim _{\lambda} \tau\left(\tilde{\iota}\left(z_{i \lambda}\right)\right)=\lim _{\lambda} \iota\left(z_{\lambda}\right)=p \quad(i=1, \cdots, n)
$$

since $\tau\left(\tilde{\imath}\left(z_{i \lambda}\right)\right)=\iota\left(z_{\lambda}\right)(i=1, \cdots, n)$. Therefore we conclude that

$$
\left\{p_{1}, \cdots, p_{n}\right\} \subset \tau^{-1}(p) .
$$

We next claim that the above inclusion relation is improper. If this is not the case, then there exists a point $q$ in $\tau^{-1}(p)-\left\{p_{1}, \cdots, p_{n}\right\}$. By the separation lemma we can find an $f$ in $H^{\infty}(\tilde{R})$ such that 


$$
\hat{f}(q) \notin\left\{\hat{f}\left(p_{1}\right), \cdots, \hat{f}\left(p_{n}\right)\right\} .
$$

Let $a_{1}, \cdots, a_{n}$ be the $a$-functions of $f$. Then by (4)

$$
(-1)^{k} \hat{a}_{k}\left(\iota\left(z_{\lambda}\right)\right)=\sum_{i_{1}<\cdots<i_{k}}^{1, \cdots, n} \hat{f}\left(\tilde{c}\left(z_{i_{1}}\right)\right) \cdots \hat{f}\left(\tilde{c}\left(z_{i_{k}}\right)\right)
$$

for each $k=1, \cdots, n$. Taking the limit with respect to $\lambda$, we have

$$
(-1)^{k} \hat{a}_{k}(p)=\sum_{i_{1}<\cdots<i_{k}}^{1, \cdots, n} \hat{f}\left(p_{i_{1}}\right) \cdots \hat{f}\left(p_{i_{k}}\right) .
$$

Therefore $S=\left\{\hat{f}\left(p_{1}\right), \cdots, \hat{f}\left(p_{n}\right)\right\}$ is the totality of solutions of the algebraic equation

$$
X^{n}+\hat{a}_{i}(p) X^{n-1}+\cdots+\hat{a}_{n-1}(p) X+\hat{a}_{n}(p)=0
$$

of degree $n$. By considering the Gelfand transform of (7) at $q$, we obtain that

$$
\hat{f}(q)^{n}+\widehat{a_{1} \circ \pi}(q) \hat{f}(q)^{n-1}+\cdots+\widehat{a_{n-1} \circ \pi}(q) \hat{f}(q)+\widehat{a_{n} \circ \pi}(q)=0 .
$$

Since $\widehat{a_{i} \circ \pi}(q)=\hat{a}_{i}(\tau(q))=\hat{a}_{i}(p) \quad(i=1, \cdots, n)$, the above identity shows that $X=\hat{f}(q)$ satisfies (12), i.e. $\hat{f}(q) \in S$, which contradicts (11). Since $p_{i} \in \bar{\tau}(\tilde{R})$ by the choice of $p_{i}(i=1, \cdots, n)$, we conclude that

$$
\tau^{-1}(p)=\left\{p_{1}, \cdots, p_{n}\right\} \subset \overline{\tilde{\imath}(\tilde{R})}
$$

which shows that $\tau^{-1}(\overline{\iota(R)}) \subset \overline{\imath(\widetilde{R})}$.

12. Finally we show that $\tilde{M}$ contains a free point if and only if $M$ contains a free point.

Suppose $p$ is a free point in $\tilde{M}$. If $\tau(p)$ belongs to $\overline{\iota(R)}$, then the second identity of $(10)$ implies that $\tau^{-1}(\tau(p)) \subset \bar{\tau}(\tilde{R})$. Since $p$ belongs to $\tau^{-1}(\tau(p))$, we see that $p$ belongs to $\bar{\tau}(\tilde{R})$ which shows that $p$ is not free. Thus $M$ contains a free point $\tau(p)$.

Conversely suppose that $q$ is a free point in $M$. Let $p$ be an arbitrary point in $\tau^{-1}(q)$. We claim that $p$ is free. If $p$ is not free, then $p$ belongs to $\tilde{\imath(\tilde{R})}$, and the first identity of $(10)$ implies that $\tau(p)$ belongs to $\overline{\iota(R)}$. Hence $q=\tau(p)$ is not free which is a contradiction. Therefore $p$ is a free point in $\tilde{M}$. 


\section{REFERENCES}

[1] L. Ahlfors, Open Riemann surfaces and extremal problems on compact subregions, Comm. Math. Helv., 24 (1950), 100-134.

[2] M. Behrens, The maximal ideal space of algebras of bounded analytic functions on infinitely connected domains, Trans. Amer. Math. Soc., 161 (1971), 359-380.

[ 3 ] L. Carleson, Interpolation by bounded analytic functions and the corona problem, Ann. of Math., 76 (1962), 547-559.

[4] T. Gamelin, Localization of the corona problem, Pacific J. Math., 34 (1970), 73-81.

[ 5 ] —-, Uniform Algebras, Prentice-Hall, 1969.

[6] — Uniform Algebras and Jensen Measures, London Math. Soc. Lecture Note Series, 32, 1978.

[ 7 ] —-Wolff's proof of the corona theorem, Israel J. Math., 37 (1980), 113-119.

[ 8 ] M. Hara, On Gamelin constants, NIT Seminar Note, 1981.

[ 9 ] - The corona problem on 2-sheeted disks, Proc. Japan Acad., 58 (1982), 256257.

[10] M. Nakai, Corona problem for Riemann surfaces of Parreau-Widom type, Pacific J. Math., 103 (1982), 103-109.

[11] W. Rudin, Real and Complex Analysis, McGraw-Hill, 1966.

[12] S. Segawa, Bounded analytic functions on unbounded covering surfaces, Pacific J. Math., 79 (1978), 183-187.

[13] H. Selberg, Ein Satz über beschränkte endlichvieldeutige analytische Funktionen, Comm. Math. Helv., 9 (1937), 104-108.

[14] C. Stanton, Bounded analytic functions on a class of open Riemann surfaces, Pacific J. Math., 59 (1975), 557-565.

[15] E. Stout, Two theorems concerning functions holomorphic on multiply connected domains, Bull. Amer. Math. Soc., 69 (1963), 527-530.

[16] H. Widom, The maximum principle for multiple valued analytic functions, Acta Math., 126 (1971), 63-82.

[17] —, $H^{p}$-sections of vector bundles over Riemann surfaces, Ann. of Math., 94 (1971), 304-324.

[18] Y. Yamamura, On the existence of bounded analytic functions, Sci. Rep. Tokyo Kyoiku Daigaku, Sect. A, 10 (1969), 88-102.

[19] O. Zariski and P. Samuel, Commutative Algebra, Vol. 1, Van Nostrand, 1958.

Department of Mathematics

Nagoya Institute of Technology

Gokiso, Showa, Nagoya 466

Japan 